\title{
COLLINEAR PHOTON EMISSION FROM THE QUARK-GLUON PLASMA: THE LIGHT-CONE PATH INTEGRAL FORMULATION
}

\author{
P. Aurenche ${ }^{1}$ and B.G. Zakharov ${ }^{2}$ \\ ${ }^{1}$ LAPTH, Université de Savoie, CNRS, 9 Chemin de Bellevue, B.P. 110, F-74941 \\ Annecy-le-Vieux Cedex, France \\ ${ }^{2}$ L.D. Landau Institute for Theoretical Physics, GSP-1, 117940, \\ Kosygina Str. 2, 117334 Moscow, Russia
}

\begin{abstract}
We give a simple physical derivation of the photon emission rate from the weakly coupled quark-gluon plasma connected with the collinear processes $q \rightarrow \gamma q$ and $q \bar{q} \rightarrow \gamma$. The analysis is based on the light-cone path integral approach to the induced radiation. Our results agree with that by Arnold, Moore and Yaffe obtained using the real-time thermal perturbation theory. It is demonstrated that the solution of the AMY integral equation is nothing but the time-integrated Green's function of the light-cone path integral approach written in the momentum representation.
\end{abstract}


1. In recent years much attention has been given to the problem of the photon radiation from the hot quark-gluon plasma (QGP). This interest is mostly motivated by the possibility of production of the QGP in the heavy-ion collisions that are under active investigation at RHIC, and will be studied soon at LHC. The thermal photon radiation is expected to be a good probe of formation of the QGP, since the photons can freely escape the plasma and give information about the plasma temperature, $T$. The photon emission rate per unit time and volume can be written as

$$
\frac{d N}{d t d V d \mathbf{k}}=-\frac{n_{B}(\omega)}{\omega(2 \pi)^{3}} \operatorname{Im} \Pi_{\mu}^{\mu}(\omega, \mathbf{k}),
$$

where $\Pi_{\mu}^{\mu}$ is the retarded photon polarization tensor, $n_{B}(\omega)=(\exp (\omega / T)-1)^{-1}$ is the photon Bose-Einstein factor. Presently the calculation of the photon polarization tensor is usually performed within the thermal perturbative Quantum Chromodynamics (pQCD) methods. The application of the pQCD to description of the QGP includes the well-known hard thermal loop (HTL) resummation [1] which is based on separation of the hard quark and gluon modes with momentum $p \sim T$ and soft collective modes with $p \sim g T$. The HTL approximation leads to the infrared finite leading order $O\left(g^{2}\right)$ contribution to the photon radiation rate connected with annihilation $(q \bar{q} \rightarrow \gamma g)$ and Compton mechanism $(q g \rightarrow \gamma q))[2]$.

The analysis of the two-loop contribution (connected with the bremsstrahlung $q \rightarrow$ $\gamma q$ and the induced annihilation $q \bar{q} \rightarrow \gamma$ physical processes) to the polarization tensor demonstrated [3] that it turns out to be of the same order in the coupling constant as the leading order result. This fact is a consequence of the collinear nature of the bremsstrahlung and induced annihilation, which leads to the divergence of their cross sections in the massless limits. In the thermal bath this divergence is regularized by the thermal quark mass $m_{q}^{t h} \sim g T$, and as a result these processes give a contribution of the same order as the Born approximation. It turns out that the higher-order ladder diagrams give the contribution of the same order as the two-loop diagrams [4]. The calculation of the ladder diagrams has been performed by Arnold, Moore and Yaffe (AMY) [5]. The AMY [5] result can be written as

$$
\begin{aligned}
\frac{d N}{d t d V d \mathbf{k}}=\frac{N_{c} \alpha_{e m} \sum_{s} q_{s}^{2}}{4 \pi^{2} k} \int_{-\infty}^{\infty} \frac{d p}{2 \pi} n_{F}(p & +k)\left[1-n_{F}(p)\right] \frac{(p+k)^{2}+p^{2}}{(p+k)^{2} p^{2}} \\
& \times \operatorname{Re} \int \frac{d \mathbf{p}_{\perp}}{(2 \pi)^{2}} \mathbf{p}_{\perp} \cdot \mathbf{f}\left(\mathbf{p}_{\perp}, p, \mathbf{k}\right),
\end{aligned}
$$

where $q_{s}$ is the charge of quark species type $s, n_{F}(p)=1 /(\exp (p / T)+1)$ is the thermal Fermi distribution, and $\mathbf{f}$ satisfies the integral equation

$$
i \delta E \mathbf{f}\left(\mathbf{p}_{\perp}, p, \mathbf{k}\right)=2 \mathbf{p}_{\perp}-g^{2} C_{F} T \int \frac{d \mathbf{q}_{\perp}}{(2 \pi)^{2}} C\left(\mathbf{q}_{\perp}\right)\left[\mathbf{f}\left(\mathbf{p}_{\perp}, p, \mathbf{k}\right)-\mathbf{f}\left(\mathbf{p}_{\perp}-\mathbf{q}_{\perp}, p, \mathbf{k}\right)\right] .
$$

Here, $C_{F}=4 / 3$ is the quark Casimir, $\delta E=\left|k\left(\mathbf{p}_{\perp}^{2}+m_{q}^{2}\right) / 2(p+k) p\right|, m_{q}$ is the asymptotic thermal quark quasiparticle mass, the kernel of the integral equation can be expressed 
via the HTL resummed gluon propagator. It can be written in a simple form as $C\left(\mathbf{q}_{\perp}\right)=$ $m_{D}^{2} / \mathbf{q}_{\perp}^{2}\left(\mathbf{q}_{\perp}^{2}+m_{D}^{2}\right)[6]$, where $m_{D}=g T\left[\left(N_{c}+N_{F} / 2\right) / 3\right]^{1 / 2}$ is the Debye mass. The $C\left(\mathbf{q}_{\perp}\right)$ includes the contribution of both the screened longitudinal gluon as well as the unscreened transverse gluon.

Physically the ladder diagrams describe the Landau-Pomeranchuk-Migdal (LPM) suppression $[7,8]$ of the collinear bremsstrahlung/annihilation. In the last decade considerable progress has been made in understanding the LPM effect in QCD for fast partons with energy $E \gg T$ propagating through the QGP. Two different approaches to the LPM effect in this regime have been developed: the BDMPS formalism $[9,10]$ and the light-cone path integral (LCPI) formalism [11, 12, 13, 14] (for a review, see $[15,16]$ ). In the LCPI approach the radiation rate is expressed via the Green's function of a two-dimensional Schrödinger equation with an imaginary potential.

In the studies $[9,10,11,12]$ the QGP has been modeled by a static system of the Debye screened color centers. In the case of the photon radiation from the thermal partons one should treat on the same footing the partons from which the photon is radiated and the target partons. In the AMY analysis [5] it was done using the real time thermal perturbation theory. In the present paper we give an alternative simple physical derivation of the AMY result within the LCPI approach. We demonstrate that in the case of the weakly coupled QGP when one can separate the hard and soft modes the treatment of the LPM suppression for the photon emission from the thermal partons can be made similarly to the case of fast partons. We show that the solution of the AMY integral equation (3) is nothing but the time-integrated Green's function of the LCPI approach written in the momentum representation.

Whether the model of the weakly coupled QGP, used in the present and AMY [5] analyses, is a reasonable approximation to the QGP which is produced in $A A$-collisions is still an open question. However, in principle, our derivation may have a broader range of applicability then the pQCD description of the QGP, if one calculates somehow the function $C\left(\mathbf{q}_{\perp}\right)$ and the quark quasiparticle mass using the non-perturbative methods, or, say, extracts them fitting the experimental data on the jet quenching.

2. We consider the radiation of the photons with $\omega \gtrsim T$ which is controlled by the dynamics of the hard quark modes in the QGP. The average energy for hard partons $\sim 3 T$. In the weakly coupled QGP these hard partons undergo typically only small angle multiple scattering due to interaction with the random gluon fields at the momentum scale $\sim g T \ll T$. The large angle scattering is a very rare process. The typical separation between the large angle scatterings is $L_{l . a .} \sim 1 / g^{4} T$. Using this scale, from the uncertainty relation $\Delta E \Delta t \sim 1$, one can obtain for the typical off-shellness of the hard modes $\delta m \sim$ $\left(T / L_{l . a .}\right)^{1 / 2} \sim g^{2} T$, which turns out to be much smaller than the thermal quasiparticle masses for quarks and gluons $m_{q, g}^{t h} \sim g T$. Note that the $L_{l . a}$. at the same time gives the scale at which the soft rescatterings generate the scattering angle about unity. It follows from the $L$-dependence of the mean squared momentum transfer $q^{2} \sim g^{4} T^{3} L$ connected with the random walk of hard partons due to small angle multiple scattering.

The above estimates show that at the space scale considerably smaller than $L_{l . a}$ the hard parton trajectories are almost straight lines slightly distorted by soft rescatterings. Thus, at $L \ll L_{l . a}$. the hard modes can be described as the quasiclassical plane waves 
propagating in the soft random gluon field. It is important that in first approximation one can neglect the statistics effects in treating the small angle scattering. Indeed, the typical space scale for the soft gluon modes $1 / g T$ is much larger than the typical separation between the hard partons $\sim 1 / T$. For this reason from the point of view of the hard parton scattering the soft gluon field can viewed as a uniform field at the scale $\sim 1 / T$. In the uniform field all hard partons of the same type scatter in the same way, and the small angle scattering leads simply to some shift of the distribution function in the momentum space. A well-known example of this phenomenon is shift of the Fermi-sphere for the strongly degenerated Fermi gas in a uniform electric field. Any statistics effects will be suppressed by some power of $g$.

The above facts on the hard parton trajectories are important from the point of view of the treatment of the $q \rightarrow \gamma q$ and $q \bar{q} \rightarrow \gamma$ processes in the pQGP. Let us first consider the photon bremsstrahlung. From the uncertainty relation one gets for the photon formation length $L_{f} \sim 2(1-x) E / x m_{q}^{2}{ }^{1}$, where $E$ is the initial quark energy, and $x=\omega / E$ is the photon fractional longitudinal momentum (we choose the $z$ axis along the initial quark momentum). For $\omega \sim E \sim T$ one obtains $L_{f} \sim 1 / g^{2} T$. Thus, one sees that $L_{f} \ll L_{l . a}$. It means that at leading order in $g$ the photon bremsstrahlung is not affected by the hard scattering. Since the statistics effects can be neglected for small angle scattering as well, the bremsstrahlung occurs in the same way as for hard partons in a soft random gluon field. The only effect of the thermal bath of hard partons is the trivial Pauli-blocking for the final quark, which, of course, must be taken into account. Due to the collinear character of the induced photon emission, in accounting for the Pauli-blocking one can take the final quark momentum collinear to that for the initial quark. In this case the contribution of the bremsstrahlung into the photon emission rate can be written as

$$
\frac{d N_{b r}}{d t d V d \mathbf{k}}=\frac{d_{b r}}{k^{2}(2 \pi)^{3}} \sum_{s} \int_{0}^{\infty} d p p^{2} n_{F}(p)\left[1-n_{F}(p-k)\right] \theta(p-k) \frac{d P_{q \rightarrow \gamma q}^{s}(p, k)}{d k d L},
$$

where $d_{b r}=4 N_{c}$ is the number of the quark and antiquark states, $d P_{q \rightarrow \gamma q}^{s}(p, k) / d k d L$ is the probability of the photon emission per unit length from a fast quark of type $s$ in the random soft gluon field without the thermal bath.

The formation length for the $q \bar{q} \rightarrow \gamma$ mechanism is also much smaller than $L_{l . a .}$. It means that the photon is produced in annihilation of almost collinear quark-antiquark pair induced by the small angle rescatterings in the soft random gluon field. The contribution to the radiation rate can be easily obtained using the detailed balance principle. For the plasma size much smaller than the photon absorption length it gives

$$
\frac{d N_{a n}}{d t d V d \mathbf{k}}=\left[1+n_{B}(k)\right]^{-1} \frac{d N_{a b s}}{d t d V d \mathbf{k}} .
$$

The photon absorption rate on the right-hand side of Eq. (5) should be evaluated for the thermolized photons. It can be written via the probability distribution per unit length for the $\gamma \rightarrow q \bar{q}$ transition $d P_{\gamma \rightarrow q \bar{q}}^{s}(k, p) / d p d L$, where $p$ is the final quark momentum, similarly

\footnotetext{
${ }^{1}$ This estimate does not account for the LPM suppression. If the radiation rate is suppressed by a factor $S_{L P M}$, the real in-medium formation length is $L_{f}^{L P M} \sim S_{L P M} L_{f}$.
} 
to the formula (4). Then, using (5) one obtains

$$
\frac{d N_{a n}}{d t d V d \mathbf{k}}=\frac{d_{a n}}{(2 \pi)^{3}} \sum_{s} \int_{0}^{\infty} d p n_{F}(p) n_{F}(k-p) \theta(k-p) \frac{d P_{\gamma \rightarrow q \bar{q}}^{s}(k, p)}{d p d L},
$$

where $k-p$ is the antiquark momentum, $d_{a n}=2$ is the number of the photon helicities. 3. The probability distributions entering (4) and (6) for the case of the random soft gluon field can be evaluated in the LCPI approach [11] similarly to the case of the static model of the QGP which has been used in [11] only for simplicity. For this reason we limit ourselves to highlighting only the aspects connected with the generalization to the arbitrary random external gluon field. The starting point of the LCPI approach is the representation of the parton wave function in the form

$$
\psi(t, \mathbf{r})=\exp (-i E \xi) \phi(z, \xi, \boldsymbol{\rho})
$$

where $\xi=t-z, \mathbf{r}=(z, \boldsymbol{\rho})$, and it is assumed that $E>>m_{q}$. To leading order in $m_{q} / E$ the z-dependence of the transverse wave function $\phi(z, \xi, \boldsymbol{\rho})$ for fixed $\xi$ is governed by the two-dimensional Schrödinger equation

$$
i \frac{\partial \phi(z, \boldsymbol{\rho})}{\partial z}=\hat{H} \phi(z, \boldsymbol{\rho})
$$

with the Hamiltonian

$$
\hat{H}=\frac{\left[\left(\mathbf{p}_{\perp}-g \mathbf{A}_{\perp}\right)^{2}+m_{q}^{2}\right]}{2 \mu_{q}}+g\left(A_{0}-A_{z}\right) .
$$

Here, the Schrödinger mass is $\mu_{q}=E, \mathbf{p}_{\perp}$ is the operator of transverse momentum, $A_{\mu}=\left(A_{0},-\mathbf{A}_{\perp},-A_{z}\right)$ is the external gluon vector potential $\left(A_{\mu}\right.$ should be understood as $\hat{\tau}_{q}^{a} A_{\mu}^{a}$, for clarity we omit the color generators). The argument $\xi$ of $\phi$ is omitted in (8), since the $\xi$ dependence emerges only via the boundary condition for $\phi$. For a random external potential without loss of generality we can set $\xi=0$. For this choice the vector potential in the Hamiltonian (9) must be taken at $x^{\mu}=(t, \boldsymbol{\rho}, z)$. Thus the Hamiltonian (9) describes the evolution of the partonic wave function along the light-cone. Eqs. (7), (8) hold for each helicity state. The photon wave function can be written in a similar way (of course, in this case the interaction and mass terms do not appear).

The matrix elements for the $q \rightarrow \gamma q$ and $\gamma \rightarrow q \bar{q}$ transitions can be written in terms of the Green's function for the Hamiltonian (9) and its photon analogue. In the LCPI approach they are written in the Feynman path integral form. The quark Green's function reads

$$
K_{q}\left(\boldsymbol{\rho}_{2}, z_{2} \mid \boldsymbol{\rho}_{1}, z_{1}\right)=\int \mathcal{D} \boldsymbol{\rho} \exp \left\{i \int d z\left[\frac{\mu_{q} \dot{\boldsymbol{\rho}}^{2}}{2}-g \dot{x}^{\mu} A_{\mu}(\boldsymbol{\rho}, z)-\frac{m_{q}^{2}}{2 \mu_{q}}\right]\right\}
$$

where $\dot{\boldsymbol{\rho}}=d \boldsymbol{\rho} / d z, \dot{x}^{\mu}=(1, \dot{\boldsymbol{\rho}}, 1)$. Since $|\dot{\boldsymbol{\rho}}| \ll 1$ one can replace $\dot{x}^{\mu}$ by $u^{\mu}=\left(1,0_{\perp}, 1\right)$. As compared with Ref. [11] where the static potential with only $A_{0}(\mathbf{r})$ nonzero component has been used, now it is replaced by $A^{-}=u^{\mu} A_{\mu}(x)$ with $x^{\mu}=(z, \boldsymbol{\rho}, z)$. 
Let us consider the $q \rightarrow \gamma q$ transition. The probability of the photon emission per unit length diagrammatically is given by the graph a of Fig. 1, where the longitudinal coordinate of one of the vertex is fixed. Note that due to the relation $-\hat{\tau}_{q}^{*}=\hat{\tau}_{\bar{q}}$ the quark Wilson lines in the path integral for quark propagators turns out to be replaced by the antiquark Wilson lines in the complex conjugate quark propagators.

Similarly to the static potential one can perform in the diagram 1a averaging over the external potential at the level of integrands. Then the interaction with the external potential turns out to be transformed into the interaction between trajectories which depends only on the relative transverse distances between the trajectories. This fact allows one to perform some path integrations analytically, and transform the graph 1a to $1 b$. (the details can be found in $[14,15,16]$ ), in which the only dynamical variable is the transverse separation between quark and photon $\boldsymbol{\rho}=\boldsymbol{\rho}_{q}-\boldsymbol{\rho}_{\gamma}$, and the antiquark is located at the center of mass of the $q \gamma$-system. The propagator describing the evolution of a spurious $q \bar{q} \gamma$-system in graph b of Fig. 1 in the path integral form reads

$$
\mathcal{K}\left(\boldsymbol{\rho}_{2}, z_{2} \mid \boldsymbol{\rho}_{1}, z_{1}\right)=\int \mathcal{D} \boldsymbol{\rho} \exp \left\{i \int d z\left[\frac{M(x) \dot{\boldsymbol{\rho}}^{2}}{2}-\frac{1}{L_{f}}\right]\right\} \Phi\left(\{\boldsymbol{\rho}\}, z_{1}, z_{2}\right),
$$

where $x=\omega / E$ is the photon fractional momentum, $M(x)=E x(1-x)$ is the reduced Schrödinger mass, $L_{f}=2 E(1-x) / x m_{q}^{2}$ is the photon formation length which comes from the mass terms in the quark propagators (10), and

$$
\Phi\left(\{\boldsymbol{\rho}\}, z_{1}, z_{2}\right)=\left\langle\left\langle\exp \left\{-i g \int d z\left[A_{q}^{-}\left(\boldsymbol{\rho}_{q}, z\right)+A_{\bar{q}}^{-}\left(\boldsymbol{\rho}_{\bar{q}}, z\right)\right]\right\}\right\rangle\right\rangle
$$

is the averaged Wilson line factor. The quark and antiquark transverse coordinate in (12) in therms of $\boldsymbol{\rho}$ are given by $\boldsymbol{\rho}_{q}=x \boldsymbol{\rho}, \boldsymbol{\rho}_{\bar{q}}=0$. For nonzero $\boldsymbol{\rho}_{1,2}$ the propagator (11) is not gauge invariant without the Wilson lines connecting the quark trajectories at $z_{1,2}$. This fact, however, is not important since in the final formula for the radiation rate this propagator only appears for the point-like initial and final states, i.e., for the configurations shown in Fig. 1b. The formula for the probability of the photon emission per unit length is similar to that for the static potential (we use here the fractional photon momentum $x$ instead of $k$ in (4))

$$
\frac{d P_{q \rightarrow \gamma q}^{s}}{d x d L}=\left.2 \operatorname{Re} \int_{-\infty}^{0} d z \hat{g}(x)\left[\mathcal{K}\left(\boldsymbol{\rho}_{1}, 0 \mid \boldsymbol{\rho}_{2}, z\right)-\mathcal{K}_{v a c}\left(\boldsymbol{\rho}_{1}, 0 \mid \boldsymbol{\rho}_{2}, z\right)\right]\right|_{\boldsymbol{\rho}_{1,2}=0}
$$

where $K_{v a c}$ is the free propagator for $\Phi=1^{2}$. The factor $\hat{g}$ in (13) is the vertex operator which accumulates the summation over the particle helicities. It is given by

$$
\begin{gathered}
\hat{g}(x)=\frac{1}{2} \sum_{\{\lambda\}} V^{*}(x,\{\lambda\}) V(x,\{\lambda\})\left(\frac{\partial}{\partial \rho_{x}}-i \lambda_{\gamma} \frac{\partial}{\partial \rho_{y}}\right)_{2}^{*} \cdot\left(\frac{\partial}{\partial \rho_{x}}-i \lambda_{\gamma} \frac{\partial}{\partial \rho_{y}}\right)_{1}, \\
V(x,\{\lambda\})=\frac{-i q_{s}}{2 M(x)} \sqrt{\frac{\alpha}{2 x}}\left[\lambda_{\gamma}(2-x)+2 \lambda_{q} x\right]
\end{gathered}
$$

\footnotetext{
${ }^{2}$ In the case of an infinite plasma the vacuum term in (13) gives zero contribution. However, it is necessary for selection of the induced radiation for a hard parton produced in the medium
} 
where $\{\lambda\}$ is the set of helicities for the $q \rightarrow \gamma q$ transition, as in (2) $q_{s}$ is the quark charge. The vertex factor (14) corresponds to the averaged over the quark color states probability, the corresponding normalization factor $1 / N_{c}$ is canceled by the $N_{c}$ which comes from the summing over the colors in the quark loop in the diagram of Fig. 1b.

Taking the advantage of the fact that the correlation radius for soft modes is much smaller than the photon formation length, the Wilson line factor $\Phi$ can be written in the exponential form

$$
\Phi\left(\{\boldsymbol{\rho}\}, z_{1}, z_{2}\right)=\exp \left[-\int_{z_{1}}^{z_{2}} d z P(x \boldsymbol{\rho}(z))\right],
$$

where, to leading order in the coupling constant, $P(\boldsymbol{\rho})$ diagrammatically is given by the graphs a, b, c of Fig. 2. The wavy lines in Fig. 2 correspond to the gluon correlator (the color indexes are omitted)

$$
G(x-y)=u_{\mu} u_{\nu}\left\langle\left\langle A^{\mu}(x) A^{\nu}(y)\right\rangle\right\rangle .
$$

The parallel straight lines in Fig. 2 show the quark trajectories along the light-cone $t=z$, and the $z$-integrations along trajectories are performed with a fixed position of one of the vertex. We neglect the contribution to the function $P(\boldsymbol{\rho})$ from the higher order diagrams, some of the diagrams of the order $g^{4}$ are shown in Figs. 2d, e, f. This approximation assumes that the interaction of hard partons with soft modes should be sufficiently small at the longitudinal scale about the Debye screening radius. One can say, that neglecting the higher order contribution to $P(\boldsymbol{\rho})$ corresponds to the dilute gas approximation for the gluon correlators. In this approximation one gets

$$
P(\boldsymbol{\rho})=g^{2} C_{F} \int_{-\infty}^{\infty} d z\left[G\left(z, 0_{\perp} z\right)-G(z, \boldsymbol{\rho}, z)\right] .
$$

Here we have taken into account the effect of the quark/antiquark color generators, which leads to appearing of the quark Casimir factor for each gluon correlator. Note that the QED analogue of (18) has been introduced in [17] in the analysis of propagation of the relativistic positronium atoms in amorphous media. One can write $P(\boldsymbol{\rho})$ as the momentum integral

$$
P(\boldsymbol{\rho})=\int \frac{d \mathbf{q}_{\perp}}{(2 \pi)^{2}}\left[1-\exp \left(i \boldsymbol{\rho} \mathbf{q}_{\perp}\right)\right] P\left(\mathbf{q}_{\perp}\right) .
$$

One can easily show that if one uses for the gluon correlators their HTL expressions the $P\left(\mathbf{q}_{\perp}\right)$ can be expressed via the function $C\left(\mathbf{q}_{\perp}\right)$ which enters (3) as

$$
P\left(\mathbf{q}_{\perp}\right)=g^{2} C_{F} T C\left(\mathbf{q}_{\perp}\right) .
$$

Note that for the static model of the QGP the function $P(\boldsymbol{\rho})$ reads

$$
P(\boldsymbol{\rho})=\frac{n \sigma_{q \bar{q}}(\rho)}{2},
$$

where $n$ is the number density of the color centers, and $\sigma_{q \bar{q}}(\rho)$ is the dipole cross section

$$
\sigma_{q \bar{q}}(\rho)=C_{T} C_{F} \alpha_{s}^{2} \int d \mathbf{q}_{\perp} \frac{\left[1-\exp \left(i \mathbf{q}_{\perp} \boldsymbol{\rho}\right)\right]}{\left(\mathbf{q}_{\perp}^{2}+m_{D}^{2}\right)^{2}} .
$$


Here $C_{T}$ is the color Casimir of the scattering center. It is interesting that numerically the $P(\boldsymbol{\rho})$ evaluated in the static model turns out to be close to that calculated with the HTL formula for $C\left(\mathbf{q}_{\perp}\right)$ in the region $\rho \lesssim 1 / m_{D}$ which is important for the radiation processes in the QGP.

For the interaction factor written in the form (16) the propagator (11) is, evidently, the Green's function for the Hamiltonian

$$
\hat{\mathcal{H}}=-\frac{1}{2 M(x)}\left(\frac{\partial}{\partial \boldsymbol{\rho}}\right)^{2}-i P(\boldsymbol{\rho} x)+\frac{1}{L_{f}} .
$$

The formulas for the radiation rate in terms of the solution of the Schrödinger equation with the Hamiltonian (23) with smooth boundary conditions which are convenient for numerical calculations can be found in $[13,18]$.

To establish the connection between the above formulas of the LCPI approach and those of AMY [5] we rewrite formula (13) in terms of the in-medium light-cone wave function of the spurious $q \bar{q} \gamma$-system $\Psi_{m}(x, \boldsymbol{\rho},\{\lambda\})$. For $\lambda_{q^{\prime}}=\lambda_{q}$ it reads

$$
\Psi_{m}(x, \boldsymbol{\rho},\{\lambda\})=\left.V(x,\{\lambda\})\left(\frac{\partial}{\partial \rho_{x}^{\prime}}-i \lambda_{\gamma} \frac{\partial}{\partial \rho_{y}^{\prime}}\right) \int_{-\infty}^{0} d z \mathcal{K}\left(\boldsymbol{\rho}, 0 \mid \boldsymbol{\rho}^{\prime}, z\right)\right|_{\boldsymbol{\rho}^{\prime}=0}
$$

The analogue of (24) in the vacuum case after $z$-integration gives the well-known light-cone wave function of the $q \gamma$ Fock component in the quark

$$
\Psi(x, \boldsymbol{\rho},\{\lambda\})=V(x,\{\lambda\}) \exp \left(-i \lambda_{\gamma} \varphi\right) m_{q} K_{1}\left(\rho m_{q} x\right),
$$

here $K_{1}$ is the Bessel function. In terms of $\Psi_{m}$ formula (13) reads

$$
\frac{d P_{q \rightarrow \gamma q}^{s}}{d x d L}=\left.\operatorname{Re} \sum_{\{\lambda\}} V^{*}(x,\{\lambda\})\left(\frac{\partial}{\partial \rho_{x}}-i \lambda_{\gamma} \frac{\partial}{\partial \rho_{y}}\right)^{*} \Psi_{m}(x, \boldsymbol{\rho},\{\lambda\})\right|_{\boldsymbol{\rho}=0} .
$$

The function $\Psi_{m}$ satisfies the integral equation

$$
\Psi_{m}(x, \boldsymbol{\rho},\{\lambda\})=\Psi(x, \boldsymbol{\rho},\{\lambda\})-\int d \boldsymbol{\rho}^{\prime} W\left(\boldsymbol{\rho}, \boldsymbol{\rho}^{\prime}\right) P\left(x \boldsymbol{\rho}^{\prime}\right) \Psi_{m}\left(x, \boldsymbol{\rho}^{\prime},\{\lambda\}\right)
$$

with

$$
W\left(\boldsymbol{\rho}, \boldsymbol{\rho}^{\prime}\right)=\int_{-\infty}^{0} d z \mathcal{K}_{v a c}\left(\boldsymbol{\rho}, 0 \mid \boldsymbol{\rho}^{\prime}, z\right)=\int_{-\infty}^{0} d z \frac{M(x)}{2 \pi i|z|} \exp \left\{i\left[\frac{M(x)\left(\boldsymbol{\rho}-\boldsymbol{\rho}^{\prime}\right)^{2}}{2|z|}-\frac{|z|}{L_{f}}\right]\right\} .
$$

In momentum representation it takes the form

$$
\begin{array}{r}
\Psi_{m}\left(x, \mathbf{p}_{\perp},\{\lambda\}\right)=V(x,\{\lambda\}) \frac{p_{x}-i \lambda_{\gamma} p_{y}}{\mathbf{p}_{\perp}^{2}+\epsilon^{2}} \\
+\frac{2 i M(x)}{\mathbf{p}_{\perp}^{2}+\epsilon^{2}} \int \frac{d \mathbf{q}_{\perp}}{(2 \pi)^{2}} P\left(\mathbf{q}_{\perp}\right)\left[\Psi_{m}\left(x, \mathbf{p}_{\perp},\{\lambda\}\right)-\Psi_{m}\left(x, \mathbf{p}_{\perp}-x \mathbf{q}_{\perp},\{\lambda\}\right)\right]
\end{array}
$$


with $\epsilon=x m_{q}$. Evidently the $\Psi_{m}$ can be written in the form

$$
\Psi_{m}\left(x, \mathbf{p}_{\perp},\{\lambda\}\right)=V(x,\{\lambda\})\left[F_{x}\left(\mathbf{p}_{\perp}\right)-i \lambda_{\gamma} F_{y}\left(\mathbf{p}_{\perp}\right)\right]
$$

where now $\mathbf{F}$ is the solution to the integral equation

$$
\mathbf{F}\left(\mathbf{p}_{\perp}\right)=\frac{\mathbf{p}_{\perp}}{\mathbf{p}_{\perp}^{2}+\epsilon^{2}}+\frac{2 i M(x)}{\mathbf{p}_{\perp}^{2}+\epsilon^{2}} \int \frac{d \mathbf{q}_{\perp}}{(2 \pi)^{2}} P\left(\mathbf{q}_{\perp}\right)\left[\mathbf{F}\left(\mathbf{p}_{\perp}\right)-\mathbf{F}\left(\mathbf{p}_{\perp}-x \mathbf{q}_{\perp}\right)\right] .
$$

Making use of (15) and (30) after summing over the helicities one can rewrite (26) in momentum representation as

$$
\frac{d P_{q \rightarrow \gamma q}^{s}}{d x d L}=\frac{2 q_{s}^{2} \alpha_{e m}\left[1+(1-x)^{2}\right]}{x M(x)} \operatorname{Im} \int \frac{d \mathbf{p}_{\perp}}{(2 \pi)^{2}} \mathbf{p}_{\perp} \cdot \mathbf{F} .
$$

Similar calculations for $\gamma \rightarrow q \bar{q}$ transition give

$$
\frac{d P_{\gamma \rightarrow q \bar{q}}^{s}}{d x d L}=\frac{2 q_{s}^{2} N_{c} \alpha_{e m}\left[x^{2}+(1-x)^{2}\right]}{M(x)} \operatorname{Im} \int \frac{d \mathbf{p}_{\perp}}{(2 \pi)^{2}} \mathbf{p}_{\perp} \cdot \mathbf{F},
$$

where now $x=E / \omega, M(x)=\omega x(1-x)$, and $\mathbf{F}$ satisfies the integral equation

$$
\mathbf{F}\left(\mathbf{p}_{\perp}\right)=\frac{\mathbf{p}_{\perp}}{\mathbf{p}_{\perp}^{2}+m_{q}^{2}}+\frac{2 i M(x)}{\mathbf{p}_{\perp}^{2}+m_{q}^{2}} \int \frac{d \mathbf{q}_{\perp}}{(2 \pi)^{2}} P\left(\mathbf{q}_{\perp}\right)\left[\mathbf{F}\left(\mathbf{p}_{\perp}\right)-\mathbf{F}\left(\mathbf{p}_{\perp}-\mathbf{q}_{\perp}\right)\right] .
$$

Substituting (32) and (33) into (4) and (6) after adding together the bremsstrahlung and annihilation contributions one obtains the total photon emission rate. It can be represented exactly in the form (2), (3) after the change of variables $\mathbf{p}_{\perp} \rightarrow x \mathbf{p}_{\perp}$ for the bremsstrahlung, if one uses the function $\mathbf{f}$ connected with $\mathbf{F}$ by the relations: $\mathbf{f}=$ $-i 4 E(1-x) \mathbf{F}$ for bremsstrahlung, and $\mathbf{f}=-i 4 \omega x(1-x) \mathbf{F}$ for annihilation contributions, respectively. The difference in the variables for bremsstrahlung is connected with the fact that we use the $z$-axis along the initial quark momentum (which is natural from the point of view of the light-cone wave function for $q \rightarrow \gamma q$ transition), and in [5] it is along the photon momentum. In the collinear approximation both the choices are equivalent.

4. The proposed derivation of the radiation rate is based on the picture of the weakly coupled plasma that is formally valid at $g \ll 1$. In reality, for the QGP produced in $A A$ collisions, when $T \lesssim(2-3) T_{c}, g \sim 1.5-2$. It was proposed that for such temperatures the plasma is a strongly coupled QGP (sQGP) [19]. Nonetheless one can expect that even for the sQGP the intuitive physical picture of photon emission will be the same, and our formulas should be a good approximation (at least for sufficiently large photon energies) if one somehow calculates $P(\boldsymbol{\rho})$ using nonperturbative methods, say, uses the lattice simulation, or AdS/QCD. What is really crucial for our calculations is the approximation of small parton scattering angles at the scale of the photon formation length. To understand the situation with this approximation one can use the results of the analysis of the RHIC data on jet quenching which give the direct information about $P(\boldsymbol{\rho})$, and allows one to estimate the $p_{\perp}$ broadening for the thermal partons. In terms of $P(\boldsymbol{\rho})$ the $L$-dependence of the $p_{\perp}$ distribution can be written as [17]

$$
I\left(\mathbf{p}_{\perp}\right)=\frac{1}{(2 \pi)^{2}} \int d \boldsymbol{\rho} \exp \left[i \mathbf{p}_{\perp} \boldsymbol{\rho}-P(\boldsymbol{\rho}) L\right] .
$$


For the purpose of the qualitative estimates one can use the model of the static Debye screened centers used in [18] for the analysis of the RHIC data on the nuclear modification factor $R_{A A}$. Using the oscillator approximation of the dipole cross section $\sigma(\rho) \approx C \rho^{2}$, one obtains $\left\langle p_{\perp}^{2}\right\rangle=2 C n L$. Evaluation of $C$ in the double gluon exchange approximation with $\alpha_{s}$ frozen at the value $\alpha_{s} \approx 0.7$ at low $Q^{2}[20,21]$ gives $C \sim 0.5$ for scattering on a quark. This approximation allows one to describe the RHIC data on the nuclear modification factor [18]. In the oscillator approximation the typical in-medium photon formation length which accounts for the LPM suppression reads $L_{f}^{L P M} \sim\left[\omega(1-x) / C n x^{2}\right]^{1 / 2}[13]$. Let us consider the final quark which has the smallest energy, at the scale $\sim L_{f}^{L P M}$ one gets $\left\langle p_{\perp}^{2}\right\rangle / p_{z}^{2} \sim 2\left[C n / \omega E^{2}(1-x)^{3}\right]^{1 / 2}$. For $\omega \sim 6 T$ and $E \sim 2 \omega$ one obtains $\left\langle p_{\perp}^{2}\right\rangle / p_{z}^{2} \sim 0.3$. With increase of $\omega$ this ratio will be even smaller. In fact, the angles will be somewhat smaller if one includes the collisional energy losses which can give the contribution about $20-30 \%$ for RHIC conditions. Thus, one sees that even though the typical angles are not very small, nonetheless, the collinear approximation seems to be not far from reality, at least for sufficiently large photon energies, which, in fact, are most interesting from the experimental point of view. In principle, the fact that the collinearity is not strongly violated for RHIC conditions does not contradict to the large value of $g$. It simply says that the qualitative estimates which just count the powers of $g$ in the situation when the exact values of the coefficients are unknown, are not very reliable.

5. In summary, we have proposed a simple alternative derivation of the photon emission rate in the pQGP using the LCPI approach [11]. The new derivation is interesting for several reasons. First, the LCPI formulation is convenient for the numerical calculations. One can use the methods previously developed for the case of fast partons $[13,18]$, which reduce the calculation of the radiation rate to solving the two-dimensional Schrödinger equation with smooth boundary conditions. Secondly, since the LCPI approach is formulated in the coordinate space, it can be helpful for clarifying the applicability limits of the formulas obtained for a uniform plasma when they are applied to the expanding finite-size plasma produced in $A A$-collisions. Say, the LCPI formulation can be used to estimate the role of the boundary effects connected with the initial stage of the QGP evolution when the radiation rate is the largest. Indeed, in this region, say, for the proper time $\tau \lesssim 1 \mathrm{fm}$, probability of the photon emission in the partonic processes may be substantially reduced due to the restriction on $|z| \lesssim \tau$ in the integral on the right-hand side of (13). This is not possible in the AMY [5] formulation in the momentum representation. We leave the phenomenological applications of our results to further publications.

\section{Acknowledgements}

This research is supported by the "Laboratoire Européen associé Physique Théorique et Matiére Condensée" (ENS-LANDAU). BGZ is grateful to the High Energy Group of the ICTP and the LAPTH for hospitality during his visits there. The work of BGZ was supported in part by the Grant RFBR 06-02-16078-a. 


\section{References}

[1] E. Braaten and R.D. Pisarski, Nucl. Phys. B337, 569 (1990); B339, 310 (1990);

J. Frenkel, J.C. Taylor, Nucl. Phys. B334, 199 (1990); B374, 156 (1992).

[2] J.I. Kapusta, P. Lichard and D. Seibert, Phys. Rev. D44, 2774 (1991); R. Baier, H.

Nakkagawa, A. Niegawa and K. Redlich, Z. Phys. C53, 433 (1992).

[3] P. Aurenche, F. Gelis, R. Kobes and H. Zaraket, Phys. Rev. D58, 085003 (1998).

[4] P. Aurenche, F. Gelis and H. Zaraket, Phys. Rev. D61, 116001 (2000).

[5] P. Arnold, G.D. Moore and L.G. Yaffe, JHEP 0111, 057 (2001); JHEP 0112, 009 (2001); JHEP 0206, 030 (2002).

[6] P. Aurenche, F. Gelis and H. Zaraket, JHEP 0205, 043 (2002).

[7] L.D. Landau and I.Ya. Pomeranchuk, Dokl. Akad. Nauk SSSR 92, 535, 735 (1953).

[8] A.B. Migdal, Phys. Rev. 103, 1811 (1956).

[9] R. Baier, Yu.L. Dokshitzer, A.H. Mueller, S. Peigne and D. Schiff, Nucl. Phys. B483, 291 (1997); B484, 265 (1997).

[10] R. Baier, Yu.L. Dokshitzer, A.H. Mueller, and D. Schiff, Nucl. Phys. B531, 403 (1998).

[11] B.G. Zakharov, JETP Lett. 63, 952 (1996).

[12] B.G. Zakharov, JETP Lett. 65, 615 (1997).

[13] B.G. Zakharov, Phys. Atom. Nucl. 61, 838 (1998).

[14] B.G. Zakharov, JETP Lett. 70, 176 (1999).

[15] R. Baier, D. Schiff, and B.G. Zakharov, Ann. Rev. Nucl. Part. 50, 37 (2000); hepph/0002198 (2000).

[16] B.G. Zakharov, Nucl. Phys. Proc. Suppl. 146, 151 (2005); hep-ph/0412177.

[17] B.G. Zakharov, Sov. J. Nucl. Phys. 46, 92 (1987).

[18] B.G. Zakharov, JETP Lett. 80, 617 (2004).

[19] E. Shuryak, Nucl. Phys. A750, 64 (2005);E. Shuryak and I. Zahed, Phys. Rev. D70, 054507 (2004).

[20] N.N. Nikolaev and B.G. Zakharov, Z. Phys. C49, 607 (1991).

[21] Yu.L. Dokshitzer, V.A. Khoze and S.I. Troyan, Phys. Rev. D53, 89 (1996). 


\section{Figures}

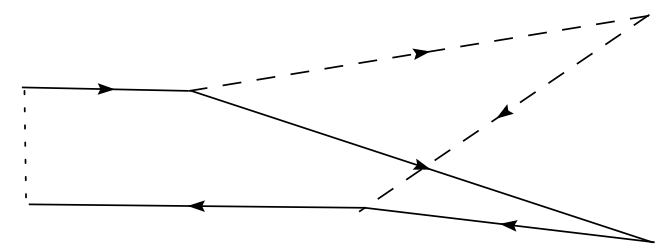

a

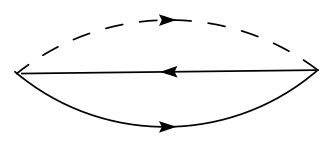

$b$

Figure 1: The diagram representation of the $x$-spectrum for $q \rightarrow \gamma q$ transition: (a) the starting form in terms of the quark and photon propagators, the left-directed lines correspond to complex conjugate propagators the vertical dotted line indicates the initial quark density matrix; (b) the final form corresponding to (13) in which the only dynamical variable is the transverse separation between photon and quark.

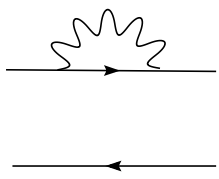

a

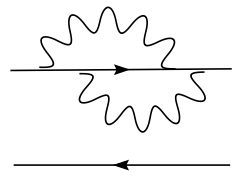

$d$

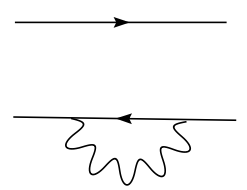

$b$

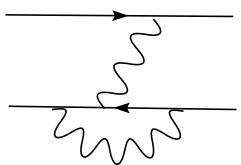

e

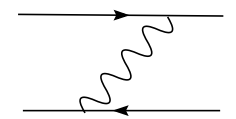

$C$

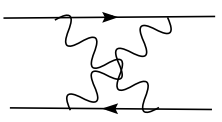

$f$

Figure 2: The diagram representation of the function $P(\boldsymbol{\rho})$ in (16): (a), (b), (c) the leading order contribution corresponding to (18); (d), (e), (f) some of the diagrams of the order of $g^{4}$. 